\title{
State-of-the-art SPECT/CT: technology, methodology and applications-defining a new role for an undervalued multimodality imaging technique
}

\author{
Thomas Beyer • Patrick Veit-Haibach
}

Received: 8 January 2014 / Accepted: 9 January 2014 / Published online: 17 April 2014

(C) Springer-Verlag Berlin Heidelberg 2014

\begin{abstract}
After more than a decade, combined multimodality imaging has now become a clinical reality. The combination of SPECT and $\mathrm{CT}$ has transformed the plain and simple bone scan into a quantitative imaging procedure. Coming from the undervalued "spots and dots" corner, the combination of functional und anatomical imaging has led to an increased acceptance among the imaging community as well as among referring physicians. The other prominent multimodality combination of this decade is that of PET and CT (called PET/CT). Being considered a quantitative combination right from the beginning, it has been used mainly in oncology imaging for staging, restaging, therapy follow-up and prognostic stratification. PET/CT has been shown to increase the average diagnostic accuracy by up to $15 \%$ [1]. It helps avoid biopsies and leads to changes in therapy planning in almost all solid tumour entities [2].

Both SPECT/CT and PET/CT have their distinctive position within the clinical and academic environment, and have forced radiologists and nuclear medicine physicians to change their working philosophies and to think in terms of hybrid imaging rather than staying with their traditional in-house modalities alone. This evolution may be even more accelerated with the advent of PET/MRI. It is widely accepted now that fusing the advantages of both radiology and nuclear medicine (or molecular imaging), be it through joined reporting by two specialists or through obtaining dual board certification, leads to a better diagnosis and thus ultimately to a benefit for the patient.
\end{abstract}

\section{T. Beyer}

Zentrum für Medizinische Physik und Biomedizinische Technik, Medizinische Universität Wien, AKHA -1090 Wien, Währinger Gürtel 18-20, 4L, Vienna, Austria

\section{P. Veit-Haibach $(\square)$}

Department Medical Radiology Diagnostic and Inverventional

Radiology Nuclear Medicine, University Hospital Zurich,

Rämistrasse 100, Zurich 8091, Switzerland

e-mail: patrick.veit-haibach@usz.ch
The development of the SPECT/CT concept was primarily driven by academia. A prototype for human use was presented as early as in the 1990s [3]. The concept then encouraged further commercial development and the first industry-built systems became available on the market in the late 1990s [4]. Today, several SPECT/CT design concepts are available commercially, and more so for preclinical use. Advanced system configurations including SPECT systems combined with CT ranging from lower-end to multislice $\mathrm{CT}$ systems, including iterative image reconstruction and advanced data processing, and have begunn to offer full quantification as exemplified by the recent introduction of such a SPECT/CT system at the annual meeting of the EANM in Lyon last year.

Originally, SPECT/CT was not considered a true competitor to radiological imaging procedures. This is in contrast to $\mathrm{PET} / \mathrm{CT}$, which rivalled classical $\mathrm{CT}$ and $\mathrm{MR}$ imaging in several indications. Today, the perception of SPECT/CT has changed. The technology has matured and more data are available to appraise its clinical role.

In this supplement of the European Journal of Nuclear Medicine and Nuclear Imaging, we bring together the perspectives and opinions of experts in physics, radiology and nuclear medicine from around the world, which show the vital potential of clinical routine and research applications in stateof-the-art SPECT/CT. All contributors are known and respected experts in their field with a broad clinical and methodological expertise. The topics in this supplement are allocated into basic and preclinical sciences, clinical diagnostic topics and special (technical as well as clinical) indications.

In the basic and preclinical section, Hutton discusses the origins of SPECT/CT, providing a technological and methodological overview from the early Anger camera to the first SPECT/CT prototypes and current state-of-the-art SPECT/CT systems [5]. As an addition, Bailey et al. provide emerging evidence that SPECT/CT can be as quantitative as PET/CT with the application of routine corrections [6]. Moving to the 
tracer side, Gnanasegaran and Ballinger in their comprehensive overview discuss tracers used routinely in SPECT/CT, as well as new tracers currently under evaluation [7]. New tracer concepts point to the steady academic interest and further illustrate the potential for increasing the range of applications of SPECT/CT. Lastly, Bernsen summarizes current preclinical applications in small-animal imaging in SPECT combined with CT and MRI [8].

The next articles in the clinical diagnostic section provide insights into the use of SPECT/CT in clinical routine cases scenarios, and they emphasize new fields in which SPECT/CT is being adopted, perhaps partly against the inclination of users who prefer MRI. Huellner and Strobel give examples demonstrating the value of SPEC/CT in imaging of the extremities, and show that SPECT/CT provides additional and therapeutically relevant information, such as in the imaging of hand and feet [9]. While such imaging is mainly in the context of chronic diseases, Scheyerer et al. also explore the possibility of integrating SPECT/CT in the subacute setting in traumatic diseases [10]. Furthermore, Abikhzer and Keidar summarize vital applications in oncology imaging, despite the increasing omnipresence of PET/CT in that area [11]. Mortensen and Gutte [12] and Gaemperli et al. [13] review two distinct procedures: the first explains the interest in pulmonary embolism imaging that is again increasing, and the latter points to the possibilities in combined cardiac perfusion/ CTA evaluation. Last but not least in this section, Nadel provides an intuitive overview on pediatric imaging protocols and selected experience with SPECT/CT [14].

The third section on special indications provides insights into the new and upcoming role of SPECT/CT in SIRT procedures. Brix et al. discuss new data on optimization and dose adaption measures to meet national diagnostic reference levels for standard situations [15]. And finally, Beckers and Hustinx provide an outline of the overarching theme of planning and conducting combined imaging procedures at the interface of nuclear medicine and radiology departments [16].

We thank all authors and contributors as well as the reviewers for their help with this timely supplement to the EJNMMI in 2014. Although it remains to be seen to what extent SPECT/CT may continue to grow in the context of the overview presented, we may speculate that the advent of commercially available quantitative methods, in particular, will place SPECT/CT in a more competitive position among clinical imaging techniques. Like PET/CT, SPECT/CT offers true multimodality imaging. By combining function and anatomy, SPECT/CT will support state-of-the-art patient management and foster academic and clinical research interests in the years to come.

\section{References}

1. Czernin J, Allen-Auerbach M, Schelbert HR. Improvements in cancer staging with PET/CT: literature-based evidence as of September 2006. J Nucl Med. 2007;48 Suppl 1:78S-88.

2. Hillner BE, Siegel BA, Shields AF, Liu D, Gareen IF, Hunt E, et al. Relationship between cancer type and impact of PET and PET/CT on intended management: findings of the national oncologic PET registry. J Nucl Med. 2008;49:1928-35. doi:10.2967/jnumed.108. 056713.

3. Lang TF, Hasegawa BH, Liew SC, Brown JK, Blankespoor SC, Reilly SM, et al. Description of a prototype emission-transmission computed tomography imaging system. J Nucl Med. 1992;33:18817.

4. Townsend DW. Multimodality imaging of structure and function. Phys Med Biol. 2008;53:R1-39. doi:10.1088/0031-9155/53/4/R01.

5. Hutton BF. The origins of SPECT and SPECT/CT. Eur J Nucl Med Mol Imaging. 2013. doi:10.1007/s00259-013-2606-5.

6. Bailey DL, Willowson KP. Quantitative SPECT/CT: SPECT joins PET as a quantitative imaging modality. Eur J Nucl Med Mol Imaging. 2013. doi:10.1007/s00259-013-2542-4.

7. Gnanasegaran G, Ballinger JR. Molecular imaging agents for SPECT (and SPECT/CT). Eur J Nucl Med Mol Imaging. 2013. doi:10.1007/ s00259-013-2643-0.

8. Bernsen MR. The role of pre-clinical SPECT in oncological and neurological research in combination with either CT or MRI. Eur J Nucl Med Mol Imaging. 2014. doi:10.1007/s00259-013-2685-3.

9. Huellner MW, Strobel K. Clinical applications of SPECT/CT in imaging the extremities. Eur J Nucl Med Mol Imaging. 2013. doi: 10.1007/s00259-013-2533-5.

10. Scheyerer MJ, Pietsch C, Zimmermann SM, Osterhoff G, Simmen HP, Werner CM. SPECT/CT for imaging of the spine and pelvis in clinical routine: a physician's perspective of the adoption of SPECT/ $\mathrm{CT}$ in a clinical setting with a focus on trauma surgery. Eur J Nucl Med Mol Imaging. 2013. doi:10.1007/s00259-013-2554-0.

11. Abikhzer G, Keidar Z. SPECT/CT and tumour imaging. Eur J Nucl Med Mol Imaging. 2013. doi:10.1007/s00259-013-2534-4.

12. Mortensen J, Gutte H. SPECT/CT and pulmonary embolism. Eur J Nucl Med Mol Imaging. 2013. doi:10.1007/s00259-013-2614-5.

13. Gaemperli O, Kaufmann P, Alkadhi H. Cardiac hybrid imaging. Eur J Nucl Med Mol Imaging. 2014. doi:10.1007/s00259-013-2566-9

14. Nadel H. SPECT/CT in pediatric patient management. Eur J Nucl Med Mol Imaging. 2014. doi:10.1007/s00259-014-2697-7.

15. Brix G, Nekolla EA, Borowski M, Nosske D. Radiation risk and protection of patients in clinical SPECT/CT. Eur J Nucl Med Mol Imaging. 2013. doi:10.1007/s00259-013-2543-3.

16. Beckers C, Hustinx R. SPECT/CT workflow and imaging protocols. Eur J Nucl Med Mol Imaging. 2013. doi:10.1007/ s00259-013-2629-y. 